\title{
Percepción de los estudiantes de una escuela de enfermería acerca del consumo de drogas lícitas e ilícitas
}

\author{
Azucena Bermúdez-Herrera ${ }^{1}$ \\ Marta Angélica lossi Silva ${ }^{2}$ \\ Elis Maria Teixeira Priotto ${ }^{3}$ \\ Julliane Messias Cordeiro Sampaio ${ }^{4}$
}

\begin{abstract}
El consumo de drogas es tan antiguo como la humanidad y siempre han existido drogas asociadas a la cultura en cada contexto histórico y social. El presente trabajo tuvo el objetivo de conocer y analizar la percepción de los estudiantes de la Escuela de Enfermería de la Universidad de Guayaquil acerca del consumo de drogas lícitas e ilícitas. El enfoque metodológico fue cualitativo, descriptivo y exploratorio. Participaron 11 estudiantes de los primeros cursos de la Escuela de Enfermería. Para obtener los datos se utilizó entrevistas individuales semiestructuradas. El análisis temático de contenido fue adoptado identificando cinco núcleos temáticos: la situación económica, la violencia intrafamiliar, la migración de los familiares cercanos, la influencia del medio que los rodea, el desconocimiento sobre el tema. Los estudiantes son conscientes de esta dura realidad que azota a la humanidad y conocer sus percepciones contribuyó para la identificación de sus necesidades y para la creación de intervenciones para el cuidado en salud, con énfasis en la promoción de la salud.
\end{abstract}

Descriptores: Drogas Ilícitas; Estudiantes de Enfermería; Promoción de la Salud; Enfermería.

\footnotetext{
${ }^{1}$ Enfermera, Profesora, Escuela de Enfermería, Universidad de Guayaquil, Ecuador. E-mail: azucena_bermudez@hotmail.com.

${ }^{2}$ Enfermera, Doctora en Enfermería. Profesor Doctor, Escola de Enfermagem de Ribeirão Preto, Universidade de São Paulo, Centro Colaborador de la OMS para el Desarrollo de la Investigación en Enfermería, SP, Brasil. E-mail: maiossi@eerp.usp.br.

${ }^{3}$ Enfermera, Estudiante de Doctorado, Escola de Enfermagem de Ribeirão Preto, Universidade de São Paulo, Centro Colaborador de la OMS para el Desarrollo de la Investigación en Enfermería, SP, Brasil. E-mail: elispalmapriotto@hotmail.com.

${ }^{4}$ Enfermera, Estudiante de Maestría, Escola de Enfermagem de Ribeirão Preto, Universidade de São Paulo, Centro Colaborador de la OMS para el Desarrollo de la Investigación en Enfermería, SP, Brasil. E-mail:enfajulliane@usp.br.
}

Correspondencia:

Marta Angélica Iossi Silva

Universidade de São Paulo. Escola de Enfermagem de Ribeirão Preto

Departamento Materno-Infantil e Saúde Pública

Av. dos Bandeirantes, 3900

Bairro: Monte Alegre

CEP: 14040-902 Ribeirão Preto, SP, Brasil

E-mail: maiossi@eerp.usp.br 


\section{Percepção dos alunos de uma escola de enfermagem acerca do consumo de drogas lícitas e ilícitas}

O consumo de drogas é tão antigo quanto a humanidade, estando associado à cultura em cada contexto histórico e social. Este estudo teve como objetivo compreender e analisar a percepção dos estudantes da Escola de Enfermagem da Universidade de Guayaquil, acerca do consumo de drogas lícitas e ilícitas. A abordagem metodológica foi qualitativa, descritiva e exploratória. Participaram 11 estudantes dos primeiros anos da Escola de Enfermagem. Para coleta dos dados, utilizaram-se entrevistas individuais semiestruturadas. A análise temática de conteúdo foi adotada, identificando-se cinco núcleos temáticos: a situação econômica, violência doméstica, migração de parentes próximos, influência do seu entorno e o desconhecimento sobre o tema. Os estudantes estão conscientes dessa realidade cruel que assola a humanidade, e conhecer suas percepções contribui para a identificação de suas necessidades e possibilidades de intervenção para o cuidado em saúde, com ênfase na promoção da saúde.

Descritores: Drogas Ilícitas; Estudantes de Enfermagem; Promoção da Saúde; Enfermagem.

\section{Nursing School Students' Perception of Legal and Illegal Drugs Consumption}

Drugs consumption is as ancient as humanity. It has always existed and is associated with culture, in its historical and social context. The aim of this research is to know and analyze the perception of students from the Nursing School at the University of Guayaquil about legal and illegal drugs consumption. The methodological approach was qualitative, descriptive and exploratory. The sample consisted of eleven first-year students from the Nursing School. Individual and semi structured interviews were used for data collection. Thematic content analysis was adopted, in which five themes were identified: The economic situation, domestic violence, migration of close relatives, influence of the media that surround us, and ignorance about the topic. With a view to enhancing awareness on this hard reality that hurts and prejudices humanity, knowing students' perceptions contributes to identify their needs and create possibilities for health care interventions, particularly health promotion.

Descriptors: Street Drugs; Students, Nursing; Health Promotion; Nursing.

\section{Introducción}

En la actualidad el principal problema de salud pública en los países desarrollados y en vías de desarrollo es el consumo de drogas. Los costos, que ello ocasiona en los ámbitos familiar, económico y social son enormes, dado que lo relacionado al uso indebido de drogas es un asunto complejo. El enfoque, el conocimiento y las estrategias son comprometidas con actividades concretas que propenden a la prevención de los niveles primario, secundario y terciario; este último implica la rehabilitación en la cual el docente es un factor socioafectivo importante para la recuperación de las/los estudiantes que requieren atención especializada.

Los jóvenes adictos a drogas como los expuestos a ellas, por estar inmersos en una sociedad en la que el problema es una realidad ya palpable, son el resultado de una crisis de valores que abarca un ámbito mayor que el 
de la individualidad y que no sólo se constituye en una problemática local sino que tiene emergencia mundial(1).

Hay que considerar 3 factores causales: el usuario, la droga y el medio en que se desarrollan e interactúan permanentemente cuando se da un caso de adicción(2).

Es innegable que hay aspectos que conciernen a la personalidad del adulto joven en cuestión, que es portador de una historia propia y por tanto única, que de algún modo explica porque de una misma familia no todos sus integrantes fueron, son o serán adictos a las drogas.

Lo enunciado nos permite detectar que la adicción no corresponde siempre a drogas ilegales y prohibidas (marihuana, cocaína y un derivado de la cocaína: "crack"), sino que diversos estudios estadísticos demuestran que el mayor consumo corresponde a drogas farmacológicas auto medicadas y al alcohol por separado o combinado con ellos.

El uso indebido de estas sustancias sicotrópicas no es propio solo de adolescentes sino de gran parte de la comunidad adulta que inconscientemente niega padecer adicciones que su medio más inmediato tolera y "legaliza".

Debíamos determinar porque de la mayoría de adultos parte la censura, el cuestionamiento y la incomprensión, y aquí nos encontramos con otra de las contradicciones, que si bien son propias del ser humano, obstaculizan la claridad de entendimiento de la problemática adulta, necesaria para brindar ayuda, aumentado en los jóvenes la credibilidad.

El consumo de drogas y alcohol en jóvenes de entre 14 y 18 años de edad ha aumentado considerablemente en el último año, según revela una encuesta realizada por el Consejo Nacional de Sustancias Estupefacientes y Sicotrópicas (CONSEP)(3); este organismo de prevención considera que el consumo de estas sustancias psicoactivas se deben a problemas familiares."Muchos jóvenes no tienen buena relación con sus padres y por ello buscan en el alcohol y las drogas una forma de escape" refiere el consultor. Lo que se consiguió con este estudio es determinar donde los chicos obtienen información sobre las drogas, el alcohol, y se destaca que es con los padres que hay que trabajar. Para el CONSEP los padres no están preparados lo suficiente como para instruir a sus hijos sobre prevención.

En cuanto a drogas lícitas el alcohol es de mayor consumo frente al tabaco, al extremo que en algunos de los países de América Latina la adicción llega a 40\% según el estudio de la Comisión Interamericana para el Control del Abuso de Drogas - CICAD - OEA ${ }^{(4)}$. La CICAD declaró que este era el primer estudio comparativo de ese tipo de la comisión y se centró principalmente en el consumo de marihuana, cocaína y pasta base como drogas ilegales, y tabaco y alcohol como legales; este fue un estudio comparativo entre Uruguay, Argentina, Chile y Ecuador.

El informe 2008 de la Oficina de las Naciones Unidas contra la Droga y el Delito - ONUDD/CICAD-OEA, señala además que estamos entre los países más fumadores

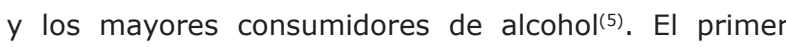
estudio comparativo sobre consumo de drogas se realizó durante 2006 y 2007 con una muestra total de 61.607 personas de 12 a 65 años, que representan a 43 millones de habitantes de seis países. Los resultados apuntan que en el caso de tabaco, $34 \%$ de los argentinos y uruguayos declararon ser común, en Chile el 44\%, 26\% en Bolivia y en Perú y en Ecuador se fuma menos, un 19\% declaró hacerlo. En cambio el consumo de alcohol presenta un patrón similar entre los países.

Considerando la importancia y complejidad del fenómeno de las drogas, las estadísticas del uso de drogas lícitas e ilícitas, las actitudes de los estudiantes universitarios y la escasez de conocimientos, el presente estudio tiene como objetivo conocer la percepción que tienen los estudiantes de la Escuela de Enfermería de la Universidad de Guayaquil acerca del consumo de drogas lícitas e ilícitas, esto nos permitirá identificar necesidades de intervención sobre núcleos encontrados y cuáles son los factores de riesgo para el consumo y los factores protectores para evitar el consumo de drogas lícitas e ilícitas.

\section{Método}

Estudio cualitativo, descriptivo y exploratorio. Las respuestas de la investigación son buscadas en el referencial del abordaje cualitativo teniendo en cuenta el objeto de estudio, el objetivo propuesto y partiendo de la comprensión de que los adolescentes son sujetos únicos que poseen una historia y una consciencia crítica con acciones permanentes de integración y significado, capaces de revelar su visión del mundo y sus percepciones ${ }^{(6)}$.

Este estudio fue aprobado por el Comité de Ética e Investigación de la Facultad de Ciencias Médicas de la Universidad de Guayaquil, con debida autorización y la firma del consentimiento informado de los sujetos del estudio, que fueron informados sobre la confidencialidad de sus respuestas, cumpliendo con los principios éticos de este tipo de investigaciones. 
La muestra del estudio fue compuesta por medio de los siguientes $\operatorname{criterios}^{(6)}$ : (a) elegir los sujetos que poseían los atributos relacionados con el estudio, (b) considerar estos en un número suficiente de modo que pudiéramos tener una recurrencia de ciertas informaciones y lograr una mayor cobertura de los sentidos y significados atribuidos al uso de drogas lícitas e ilícitas; (c) considerar la posibilidad de adiciones sucesivas de los sujetos, hasta que fue posible un debate denso de preguntas de investigación.

Con esto, no buscamos una representación numérica, sino un tema más profundo considerando la representatividad de la muestra y la capacidad de reflejar con profundidad la totalidad del fenómeno del consumo de drogas en sus múltiples dimensiones, frente a las preguntas de investigación y las referencias teóricas.

Dicho esto, el número final de las entrevistas se obtuvo mediante el criterio de la repetición y la saturación de los sentidos presentes en los discursos de los estudiantes, o sea en el conjunto de las informaciones es posible valorizar e interpretar las diferencias, o en contraposición, lo que se repetía(6-7). En la investigación cualitativa, la saturación teórica es considera un proceso continuo de análisis de datos, llevadas a cabo desde el inicio del proceso de recolección y definido como la suspensión de la inclusión de nuevos sujetos de investigación cuando los datos presentan alguna redundancia o repetición frente a las cuestiones principales y no más contribuye significativamente a la mejora de la reflexión teórica basada en los datos que están siendo recogidos(7).

La recolección de los dados se realizó a partir de entrevistas individuales, de manera individual, con los siguientes criterios de inclusión: estudiantes regularmente matriculados en el año lectivo 2010, según referencia de la dirección administrativa de la universidad; con más de 18 años y que consintiesen libremente en participar del estudio.

Así la población fue constituida por 11 estudiantes de primero, segundo y tercer cursos de la Escuela de Enfermería de la Universidad de Guayaquil.

Las entrevistas se hicieron a partir de un guión predefinido con 6 ítems compuesto de las siguientes preguntas: ¿En su opinión, que factores influyen para que los jóvenes consuman drogas? ¿Conoce que existen instituciones o Comités destinados a realizar acciones para la prevención del consumo de drogas? ¿Qué piensa sobre este trabajo en el tipo de acciones? Diga lo que piensa sobre cuáles son los factores de riesgo para el consumo de drogas lícitas e ilícitas, y ¿Las políticas económica y social del país influyen en el consumo de drogas?
La información fue recogida en grabadoras y transcrita literalmente y con exactitud, vigilando el control de calidad de los contenidos. Para garantizar el carácter sigiloso de la información de los sujetos, las entrevistas fueron codificadas por letras donde "E1" se refiere a la entrevista 1 , "E2" se refiere a la entrevista 2 y así sucesivamente. En el análisis, organizamos sistemáticamente los datos empíricos recogidos en las entrevistas, basado en las orientaciones para análisis de contenido, modalidad temática(6,8).

\section{Resultados y discusión}

El proceso de análisis cualitativo de los datos permitió identificar los siguientes núcleos de sentido: la situación económica, la violencia intrafamiliar, la migración o disfunción de familiares cercanos, la influencia del medio que los rodea y el desconocimiento sobre el tema.

La información obtenida en las entrevistas fue transcrita y trabajada con base en el análisis de contenidos $^{(7)}$, siguiendo las fases de categorización y clasificación, la exploración del material de las entrevistas se realizó mediante el proceso de "escuchar" y "re escuchar" para hacer el análisis temático y descubrir los "núcleos del sentido" y utilizar las unidades de contexto como ordenadores de análisis de contenido, pasando al proceso de análisis e interpretación e información de las significaciones que las respuestas de los estudiantes ofrecieron.

\section{La situación económica}

Los participantes del estudio informaron que la difícil situación económica es uno de los factores de riesgo para el consumo de drogas:

La situación económica del país influye en los hogares de escasos recursos económicos, por el desempleo y la emigración (...) (E2)

No hay una política económica y social que prevenga el consumo de drogas, y si existen, las leyes son débiles y no se conocen (E4)

Los jóvenes de estratos socio económicos bajos por su propia condición roban enseres o los pocos electrodomésticos de su hogar para saciar sus vicios de las drogas (E6).

De acuerdo a los expertos en economía: "Pese a que los voceros oficiales se niegan a admitirlo, y cambian criterios y conceptos según los intereses políticos coyunturales, cada vez es más evidente que el país corre el riesgo de una deflación con todas las consecuencias que acarrea esta situación. Si bien el precio del petróleo 
está convaleciendo eso no garantiza una recuperación macroeconómica e inmediata del país"(9).

Analizando lo que expresan los estudiantes y los expertos, la economía del país se ve afectada, motivo por el cual el desempleo y la migración han aumentado; además, otro de los elementos que ha sustentado en los últimos años la economía nacional son los recursos enviados desde el exterior por los emigrantes, pero a raíz de la crisis mundial que empezó a fines del 2008 esa situación cambió de manera radical.

\section{La violencia intrafamiliar}

Con respecto a este núcleo de sentido las estudiantes expresaron:

Que sus padres cuando llegaban ebrios golpeaban a sus madres y ellas se asustaban o les pegaban con látigos sin ningún motivo (E3).

En los hogares no hay respeto, consideración y tolerancia para con sus hijos, los padres agreden físicamente $y$ psicológicamente a sus hijos (E4).

Por la violencia y la incomprensión que existen en los hogares, los jóvenes buscan en las drogas su escape, su poderío (E5).

Es importante destacar que una agresión no permite suponer que exista violencia intrafamiliar, para que se pueda hablar de violencia intrafamiliar debe existir un abuso, maltrato o abandono permanente, repetido y habitual(10). Por lo tanto, la familia es el primer y más intenso agente de socialización, determinando que estímulos sociales serán presentados al niño; así este aspecto puede representar tanto un factor de riesgo como de protección para el consumo de drogas y para la violencia(10-11). Como se puede apreciar, los estudiantes expresan temor y miedo al presenciar situaciones violentas entre sus padres, la familia es el núcleo más importante en la formación de patrones de conducta en los niños, las que pueden ser positivas o negativas, en estas últimas se encuentran la violencia entre la pareja y el maltrato infantil.

En consecuencia de la violencia entre la pareja y el maltrato infantil, que son tipos de violencia intrafamiliar, los niños y adolescentes son conducidos a refugiarse en los "amigos" y son presas fáciles para consumir drogas.

Es importante apuntar que: la dificultad económica; la violencia intrafamiliar, la soledad y el alejamiento de los padres, pueden constituirse en factores protectores, por otro lado, también estos factores pueden motivar el inicio del consumo de drogas ${ }^{(11)}$.

\section{La emigración o disfunción de familiares cercanos}

Las/los estudiantes manifestaron:

Que la emigración de familiares cercanos influye en el consumo de drogas, porque los adolescentes quedan al cuidado de los tíos o abuelos y ellos no ejercen ningún control, y buscan en sus "pares" apoyo y estos los conducen al consumo de drogas lícitas e ilícitas (E1).

Muchos padres han salido a otros países especialmente a España, sus hijos se quedan con tíos o abuelos, no hay por lo tanto la figura paterna y buscan cariño y protección en sus amigos del barrio (E7).

La migración de sus padres, si ha afectado a los chicos de mi barrio, algunos fuman marihuana, beben cerveza, porque no hay la autoridad paterna. Algunos están perdidos en las drogas (E9).

Con respecto a la emigración de los ecuatorianos es posible apuntar que los niveles de emigración de ellos han ido creciendo año a año, y hemos llegado a un punto tan extremo que hoy en día el Ecuador depende económicamente de las remesas enviadas por dichos emigrantes. La principal causa de la emigración en el Ecuador es el decreciente estándar de vida y este tema se ha convertido de vital importancia en las relaciones políticas y sociales de la nación(11).

Por lo tanto, la emigración tiene sus factores negativos, por ejemplo el consumo drogas lícitas e ilícitas, siendo esto reconocido por los estudiantes; además existe el dolor del desprendimiento de los familiares afectados y sus consecuencias en el hogar. Pero por otro lado, este sacrificio tiene sus recompensas en cuanto a experiencias de vida y sobre todo múltiples beneficios y bienestar en términos económicos para las familiares.

\section{La influencia del medio que los rodea}

La falta de comunicación real de los hijos con sus padres, hace que los hijos se distancien poco a poco del entorno familiar, al no encontrar allí respuestas para sus problemas. La falta de dedicación de los padres a la educación de los hijos o el deficiente control, las actitudes de sobreprotección o las actitudes rígidas son factores desencadenantes que se supone produzcan situaciones de riesgo en la familia(3).

Además de la falta de apoyo familiar también es consecuencia de la poca comunicación o dialogo en la familia (E8).

En el medio en que se desenvuelven hay maltratos, (golpes, gritos, insultos) y los obligan a buscar en los pares el refugio $y$ encuentra en las drogas el alivio a sus frustraciones (E9). 
La discriminación, el abandono, la desorganización de la familia y los antecedentes familiares de consumo alcohol y drogas (E10).

La falta de alternativas en el tiempo de ocio de los jóvenes se traduce en grandes dificultades para que éstos no se sientan inclinados a pasar el tiempo libre en bares y discotecas. El agravamiento de una situación personal es bastante común en los antecedentes históricos del comienzo del consumo de drogas en los jóvenes. Las situaciones personales de alto riesgo como embarazos en adolescentes y la pérdida de trabajo, se consideran como causas del inicio de consumo de drogas ${ }^{(12)}$.

Analizando lo que manifiestan los estudiantes, el entorno familiar en el que existe maltrato los conducen a buscar en las drogas su refugio; y estamos de acuerdo con el consultor del CONSEP en que no hay alternativas para los jóvenes en su tiempo libre y esto los conduce frecuentar lugares no recomendables ${ }^{(3)}$.

\section{El desconocimiento sobre el tema}

La falta de conocimientos sobre la existencia instituciones que se dedican a la prevención del consumo de drogas lícitas e ilícitas, es evidente en la mayoría de las estudiantes: No conozco instituciones que ayuden a las adolescentes en la prevención del consumo de drogas lícitas e ilícitas (E4). Hemos escuchado que existe el CONSEP o si existen instituciones son centros de represión, pero no de prevención (E6).

Realizando el análisis de estos datos podemos confirmar que hay desconocimiento de las instituciones que los podrían ayudar en una situación de conflicto y también que las normas de conducta y valores son las que sustentan el hogar y la sociedad.

La disfunción familiar está caracterizada por: patrones negativos de educación y crianza, actitud negativa hacia los familiares, sistema familiar en crisis o en escenario disfuncional enmarcado por el abuso de alcohol y otras drogas en el hogar. La disfunción familiar ha sido asociada a los primeros consumos de los adolescentes $^{(13)}$.

\section{Conclusiones}

El estudio responde a los problemas y necesidades sentidas por los estudiantes de la Escuela de Enfermería de la Universidad de Guayaquil, pues se enfatizan las acciones preventivas y de promoción de la salud, pilares fundamentales de la atención primaria de salud y de los problemas de salud de alta prevalencia.
El análisis permitió comprender que los estudiantes de la Escuela de Enfermería de la Universidad de Guayaquil perciben la migración, la desintegración familiar y la violencia intrafamiliar como uno de los mayores problemas y coinciden en que la situación socioeconómica en que se desenvuelve el país (desempleo, consumismo, violencia y migración, siendo este último el más frecuente e importante) tiene como consecuencia la carencia de afecto y amor, ocasionando en ellos una formación sin valores e inestabilidad emocional; así, encuentran en las drogas el alivio a sus angustias, frustraciones y miedos, sintiéndose más capaces para enfrentar los desafíos de la vida. La desintegración familiar es un factor de riesgo que incide en todos los demás.

Los estudiantes también consideran que influye, en el fenómeno de las drogas, el medio en que se desenvuelven, la comunicación interpersonal, el maltrato y la baja autoestima.

Ante lo expuesto esta investigación obtuvo las respuestas a muchas inquietudes y una aproximación mayor en esta temática, en el sentido de aclarar aspectos importantes del fenómeno del consumo de drogas lícitas e ilícitas, y así tener una participación más efectiva de enfermería a nivel preventivo con los estudiantes.

\section{Recomendaciones}

De acuerdo a los resultados obtenidos y a las conclusiones en el presente trabajo de investigación, planteamos las siguientes recomendaciones:

Considerando que los estudiantes de primero, segundo y tercer cursos de la Carrera de Enfermería, realizan sus prácticas comunitarias en los Centros de Salud del Ministerio de Salud Pública, sería estratégico que organicen y ejecuten acciones de Enfermería dirigidas a los niños y adolescentes en los colegios de esas áreas urbanas-marginales, desarrollando programas preventivos dirigidos a los padres y profesores, con el objetivo de disminuir o evitar el consumo de drogas lícitas e ilícitas. Los programas serian fundamentados en la teoría del aprendizaje social, que establece que el hombre aprende y adquiere experiencia observando las consecuencias de su entorno y las experiencia de las personas a su alrededor; la teoría también sostiene que los pensamientos, la connivencia, las creencias y expectativas son parte de un proceso de refuerzo. Este proceso ocurre a través de la observación de su propio comportamiento y la conducta de otros, la obtención de información pertinente puede ayudar a desarrollar 
hipótesis sobre las probables consecuencias de producir ese comportamiento en el futuro.

Con los programas preventivos se pretende, por una parte discutir conductas posibles y correctas, y por otra ayudar a contrarrestar los efectos negativos del modelo que está presente a lo largo de nuestro amplio sistema social. Se puede abordar temáticas sobre: drogas lícitas e ilícitas, comunicación con los hijos, crecimiento y desarrollo del adolescente y fortalecimiento de la familia.

De esta manera los padres y profesores adquieren conocimientos para poder enfrentar este problema social que cada día se torna más complejo.

\section{Agradecimientos}

Agradecemos a la Comisión Interamericana para el Control del Abuso de Drogas/CICAD de la Secretaria de Seguridad Multidimensional/SSM de la Organización de los Estados Americanos/OEA, la Secretaria Nacional de Políticas sobre Drogas/SENAD do Gabinete de Seguridad Institucional/Brasil, la Escuela de Enfermería de Ribeirao Preto de la Universidad de Sao Paulo y Centro Colaborador de la Organización Mundial de la Salud para el Desarrollo de la Investigación en Enfermería, la población representada en los estudios de investigación, bien como a las autoridades de las universidades representadas por los participantes del Programa En-Line de Especialización en Investigación sobre el Fenómeno de las Drogas, periodos 2006, 2007, 2008 y 2009.

\section{Referencias}

1. Pons J, Borjano E. El consumo abusivo del alcohol en la adolescencia: un modelo explicativo desde la sicología social. Madrid - España. [Internet]. 1999. [acesso 2 ago 2009]. Disponível em: http://www.papelesdelpsicologo. es/vernumero. asp ?id $=1051$.

2. Iglesias EB. Bases conceptuales de la prevención de los drogodependientes. Madrid. Ministerio del Interior, Delegación del Gobierno para el Plan Nacional sobre Drogas, Secretaria General Técnica [Internet]. 2002. [acesso 2 ago 2009]. 480 p. Disponível em: http:// www.pnsd.msc.es/Categoria2/publica/pdf/Bases_ cientificas.pdf

3. Consejo Nacional de Control de Sustancias Estupefacientes y Psicotrópicas, Observatorio Ecuatoriano de las Drogas, Comisión Interamericana contra el Abuso de Drogas, Oficina de las Naciones Unidas Contra la Droga y el Delito. Segunda Encuesta Nacional a estudiantes a de educación media sobre consumo de drogas, 2005 [Internet]. Quito: Naciones Unidas, Observatorio Ecuatoriano de drogas; 2006. [acesso 2 ago 2009]. 28 p. Disponível em: http://www. cnna.gov.ec/_upload/file473_Resumen_Ejecutivo_\%20 ENCUESTA\%20ESTUDIENTES\%282005\%29.pdf

4. Comisión Interamericana para el Control del abuso de drogas: CICAD - Organización de los Estados Americanos: OEA. [acesso 2 set 2010]. Disponível em: www.comunidadandina.org/publicaestud/

5. Oetzel R. Tendencias de la cooperación internacional al desarrollo. Quito 2008. [acesso 2 set 2010]. Disponível em:https://www.cooperacion_suiza_admin.ch/ecuador/ resoruces/resource_es_181438.pdf

6. Minayo MCS. O desafio do conhecimento: pesquisa qualitativa em saúde. São Paulo: HUCITEC; 2006.

7. Fontanella BJB, Ricas J, Turato ER. Amostragem por saturação em pesquisas qualitativas em saúde: contribuições teóricas. Cad. Saúde Pública. 2008;24(1):17-27.

8. Bardin L. Análise de conteúdo. 3 ed. Lisboa: Edições $70 ; 2004$.

9. Economía y Finanzas. Editorial: "Ecuador preocupante situación económica". Diario El Comercio de Quito, Ecuador; 17 ago 2009. p. 2.

10. Bolivar XSC. Tipos de violencia intrafamiliar. Chile 2009. [acesso 2 set 2010]. Disponível em: http://www. ecovisiones.cl/informacion/tiposdeviolencia.htm

11. Riofrío GR, Nascimento LC. Consumo de drogas en los jóvenes de la ciudad de Guayaquil, Ecuador. Rev. Latino-Am. Enfermagem. 2010;18 (no esp):598-605.

12. Vergara A. Economía participativa: una brújula para el nuevo Ecuador. Revista Observatorio de la Economía Latinoamericana; n.68, Octubre 2006. [acesso 24 nov 2010]. Disponível em: http://www.eumed.net/cursecon/ ecolat/ec/

13. Consejo Nacional de Control de Sustancias estupefacientes y Psicotrópicas, CONSEP. (EQ) Guías preventivas para jóvenes, padres de familia como ayudar a una persona que consume drogas, Quito, Ecuador, 2004. Quito: CONSEP; 2004. 